\title{
Ventricular tachycardia of left bundle branch block configuration in patients with isolated right ventricular dilatation Clinical and electrophysiological features
}

\author{
EDWARD ROWLAND, WILLIAM J MCKENNA,† DECLAN SUGRUE, ROBIN BARCLAY, \\ RODNEY A FOALE, DENNIS M KRIKLER
}

From the Division of Cardiovascular Disease, Royal Postgraduate Medical School, Hammersmith Hospital, London

SUMMARY Electrophysiological studies showed ventricular tachycardia in five patients (four male, one female) with isolated right ventricular dilatation. All had been asymptomatic before the onset of palpitation which had developed in adolescence or early adult life. Tachycardia had been associated with syncope in four patients, and three had been resuscitated from ventricular fibrillation before investigation. The electrocardiogram during ventricular tachycardia showed a left bundle branch block pattern, and endocardial mapping at electrophysiological study confirmed the right ventricular origin. The presenting tachycardia could be induced in all patients by programmed stimulation, and in three patients ventricular tachycardia of differing configuration could be induced, but the right ventricular origin and left bundle branch block pattern were maintained. In two patients ventricular tachycardia degenerated into ventricular fibrillation. Cineangiography, cross sectional echocardiography, and multigated radionuclide angiography confirmed the dilated abnormal right ventricle while indicating that left ventricular function was normal. On resting electrocardiograms $\mathrm{T}$ wave inversion over the right precordial leads was the sole abnormality. There were no signs of right heart failure and exercise tolerance was normal. Four patients have received maintenance treatment with antiarrhythmic drugs, and one had undergone operative mapping and ablative surgery.

Thus ventricular tachycardia complicating right ventricular dilatation may be associated with serious symptoms and ventricular electrical instability; and in adults it may be suspected on clinical grounds by inverted $\mathrm{T}$ waves in the right precordial leads.

Ventricular tachycardia may occur in the context of organic heart disease but may also be present when cardiac structure and function are apparently normal. ${ }^{2}$ The QRS morphology of both ventricular extrasystoles ${ }^{3}$ and ventricular tachycardia ${ }^{4}$ has been proposed as a method of discrimination between right and left ventricular origin. Although electrophysiological mapping has shown that attribution on the basis of bundle branch block morphology is not always valid, 5 endocardial mapping-particularly intraoperatively - can localise the ventricular origin of ventricular tachycardia. ${ }^{6}$ The observation that ventricular tachycardia arising on the right side may be

* Research Fellow of the Medical Research Council of Canada. †Present address: Mayo Clinic, Rochester, Minnesota, USA.

Accepted for publication 9 August 1983 found in the absence of organic heart disease and that left sided arrhythmias are frequently associated with coronary or other heart disease led some investigators to ascribe a more benign prognosis to ventricular tachycardia arising from the right ventricle. ${ }^{478} \mathrm{~A}$ comparison of patients with chronic recurrent left and right ventricular tachycardia suggested that organic heart disease was unusual when tachycardia arose from the right side, although right ventricular angiography had not been performed. 4 Fontaine et al. recognised a condition in which there is apparent dysplasia of the right ventricle and which is frequently complicated by ventricular tachycardia arising from the right side. ${ }^{9}$ Two recent studies have drawn attention to the importance of considering organic disease of the right ventricle (and performing thorough investigation of the right and left ventricles) in patients 
who present with right sided ventricular tachycardia. ${ }^{1011}$ Reiter et al. elaborated on arrhythmogenic right ventricular dysplasia and other conditions which compose the "clinical spectrum of ventricular tachycardia with right bundle branch block morphology." 10

We report our experience in five patients with ventricular tachycardia of left bundle branch block configuration in whom the right ventricle was shown to be abnormal in structure and function and who underwent electrophysiological study and subsequent antiarrhythmic treatment.

\section{Patients and methods}

Five patients referred with palpitation were included in the study. Two patients in this series are also reported elsewhere. ${ }^{12}$ Ventricular tachycardia was diagnosed initially on electrocardiographic criteria when atrioventricular dissociation, atrial capture, or fusion complexes were identified and subsequently confirmed during intracardiac electrophysiological study. Left bundle branch morphology was diagnosed when the QRS complex suggested left bundle branch block aberration, exceeding $120 \mathrm{~ms}$ and having an rS appearance in lead V1. A clinical history was obtained, physical examination performed, and routine haematological indices measured. Serious symptoms were defined as syncope or cardiac arrest in association with the tachycardia. No patient had had a previous myocardial infarction.

Resting electrocardiograms were recorded at normal standardisation and also at a gain of $0.5 \mathrm{mV} / \mathrm{cm}$ at least one month after an episode of tachycardia and when the patient was not receiving any antiarrhythmic medication. Echocardiography was performed in each case using cross sectional and $M$ mode techniques. Particular attention was given to evaluating right and left ventricular cavity size from parasternal, apical, and subcostal transducer positions. ${ }^{13}$ All patients also underwent left and right heart ${ }^{14}$ catheterisation and cineangiography. In two patients coronary angiography was also performed because of atypical chest pain either associated with their palpitation or occurring independently. In addition multigated radionuclide angiograms of both the left ventricle (using technetium- $-99^{\mathrm{m}}$ ) and right ventricle (using krypton- $81^{\mathrm{m}}$ ) were performed according to our usual techniques. ${ }^{15}$ Standard exercise test using a modified Bruce protocol was performed in each patient.

Intracardiac electrophysiological study was performed in all patients. After informed consent had been obtained and premedication with diazepam given pacing and recording electrodes were introduced percutaneously from the right femoral and left subclavian veins and positioned within the heart. Pre- liminary investigation involved recording from high right atrium, coronary sinus (to record left atrial and left ventricular potentials), bundle of $\mathrm{His}$, and right ventricular apex. With this arrangement anterograde and retrograde patterns of atrioventricular conduction were studied according to our usual protocol.16 Where there was intact retrograde conduction the pattern of retrograde atrial depolarisation was mapped and atrial and ventricular stimulation performed in an attempt to induce sustained atrioventricular junctional re-entry tachycardia. In the patient in whom sustained atrioventricular re-entry tachycardia was induced atrial mapping during the arrhythmia was performed to localise the retrograde pathway. The protocol for induction of ventricular tachycardia initially involved the transfer of the right atrial electrode wire to the right ventricular cavity for mapping activation sequences during tachycardia; it was also used, when necessary, for stimulation at sites other than the right ventricular apex. Ventricular tachycardia occurred spontaneously or was induced during programmed ventricular stimulation using stimuli of twice diastolic threshold and $2 \mathrm{~ms}$ duration. Single and then double ventricular stimuli were introduced with increasing prematurity at one or more pacing rates until tachycardia was initiated. In one patient triple ventricular premature stimuli were required to induce sustained ventricular tachycardia. Termination of tachycardia was attempted by either single or double premature stimuli or overdrive pacing; if ventricular flutter or fibrillation occurred immediate DC cardioversion was performed. Left ventricular mapping was achieved by the introduction of an electrode wire via the right femoral artery into the left ventricle.

After electrophysiological study appropriate antiarrhythmic treatment was started, and in one patient in whom available drugs failed to control arrhythmia operative mapping and ablative surgery were performed.

\section{Results}

\section{CLINICAL DATA}

Five patients had ventricular tachycardia of left bundle branch morphology and abnormal right ventricular structure and function. The table shows the clinical details. There was a male preponderance and the mean age at onset of symptoms was 19 years. In all patients episodes of palpitation were particularly associated with exertion occurring either during or on recovery from exercise. Four patients had previously experienced syncope during one or more episodes of tachycardia, and three of them had been resuscitated from ventricular fibrillation before referral. There was no family history of either palpitation or sudden death. 
Table Clinical data and results of investigations in five patients with isolated right ventricular dilatation

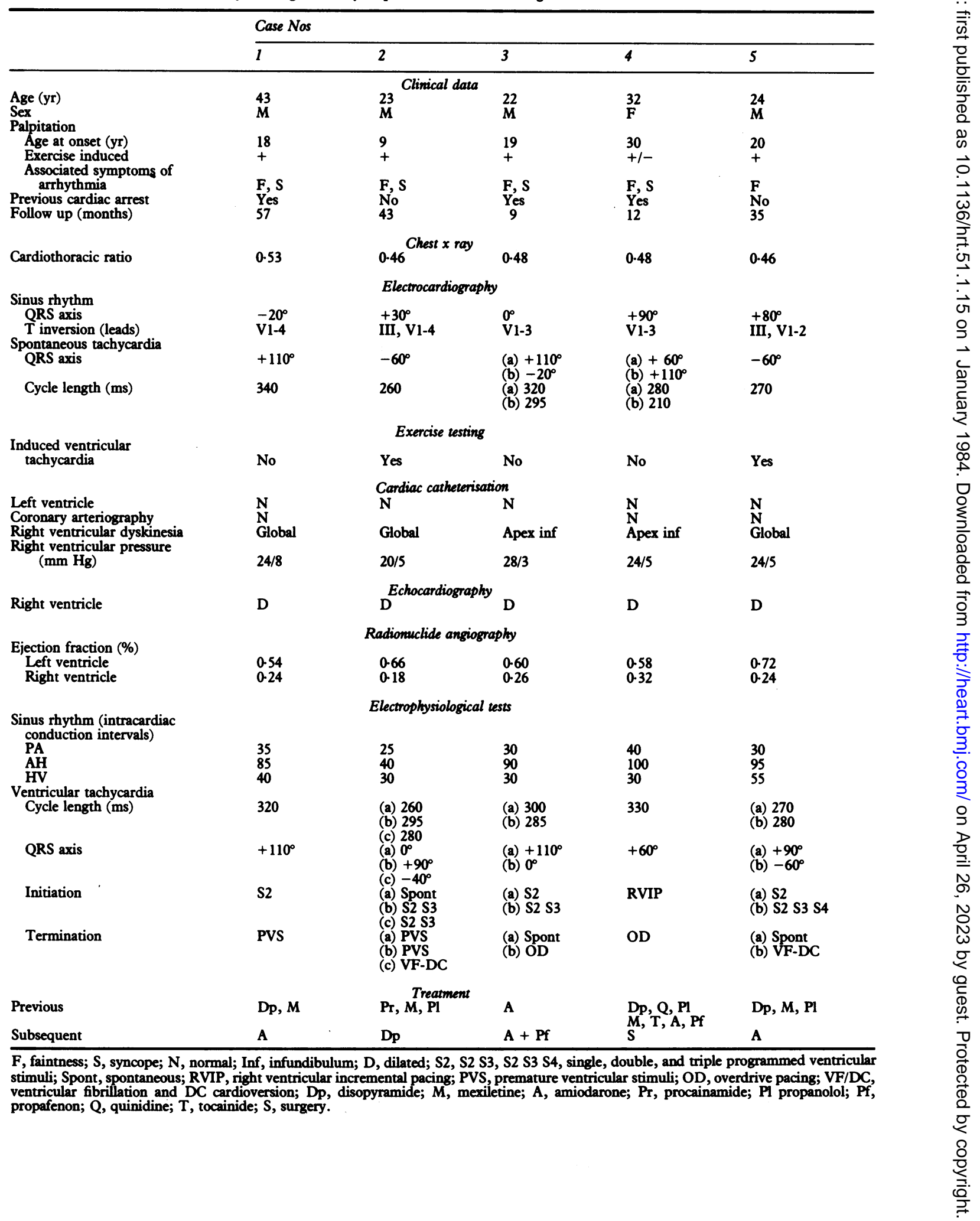




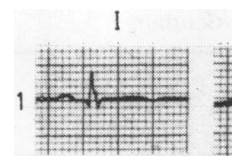

III

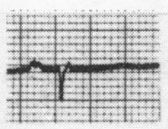

$2 \frac{1}{12}$
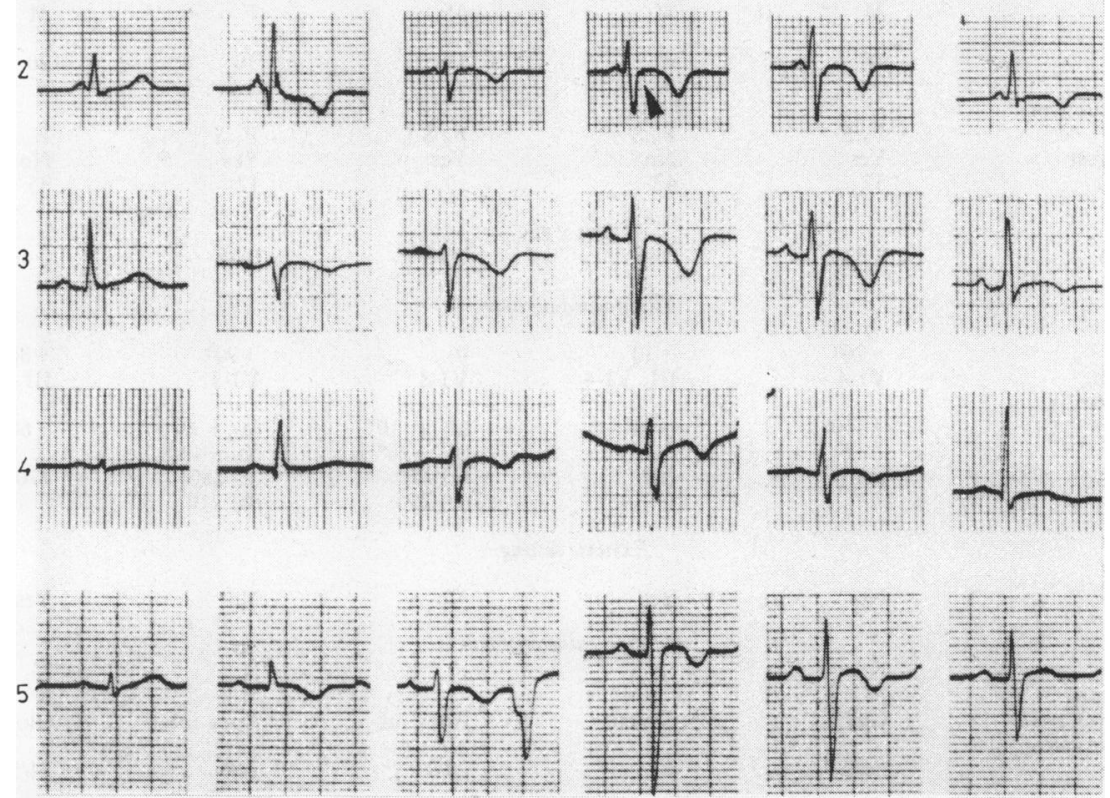

Fig. 1 Standard electrocardiograms from leads. I, III, VI-4 from each of the five patients. In each case there was $T$ wave inversion in leads V1-3 and in three cases the inversion extended to V4. Typical epsilon potentials, seen in cases 1 and 2, are arrowed.

None of the patients had abnormalities on physical examination, and none had presented with right heart failure. In one patient cardiomegaly was evident on chest $x$-ray films (Table).

\section{ELECTROCARDIOGRAPHY}

The uniform feature on electrocardiography was $T$ wave inversion in the right precordial leads (Fig. 1): all patients had $T$ wave inversion in leads V1-3, while in three this repolarisation abnormality extended to lead V4. Three patients also had $T$ wave inversion in lead III. The QRS axis was normal in all patients, and no patient showed atrioventricular or intraventricular block. In two patients (cases 1 and 2) epsilon potentials ${ }^{17}$ were evident on standard gain electrocardiography and, as with the repolarisation changes, were seen in the right precordial leads. The PR interval was short $(0.1 \mathrm{~s})$ in the patient (case 2$)$ who had atrioventricular nodal re-entry tachycardia. Electrocardiograms recorded during episodes of spontaneous tachycardia that had occurred before investigation were available in all patients (Table 1 ). In each case there were recordings of tachycardia that showed wide QRS complexes (120-160 ms, mean $136 \mathrm{~ms})$ with a left bundle branch block morphology. In two patients recordings from separate episodes were available that showed two different frontal QRS axes, although in each case the left bundle branch block configuration was still retained. Of the seven patterns of tachycardia, three showed leftward orientation of the $Q R S$ axis $\left(-20^{\circ}\right.$ to $\left.-60^{\circ}\right)$ (Fig. 2), the remainder being directed inferiorly $\left(+60^{\circ}\right.$ to $\left.+110^{\circ}\right)$. One patient showed both narrow and wide $Q R S$ complexes during different episodes of tachycardia and was shown to have atrioventricular nodal re-entry tachycardia as well as ventricular tachycardia.

During ambulatory electrocardiographic monitoring before electrophysiological study ventricular tachycardia and the subsequent degeneration into ventricular fibrillation were documented (Fig. 3). Two patients developed ventricular tachycardia on exercise testing, which terminated spontaneously in each case; exercise tolerance in the others was normal. 


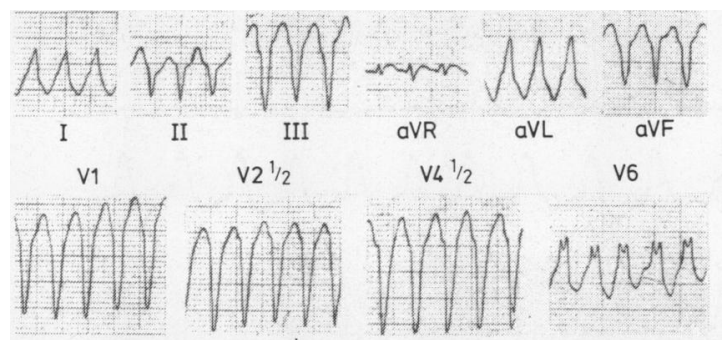

Fig. 2 Selected leads from a 12 lead electrocardiogram (case 2) taken during ventricular tachycardia showing left bundle branch block configuration and leftward frontal axis. Leads V2 and V4 were recorded at half-standardisation (paper speed $25 \mathrm{~mm} / \mathrm{s}$ ).

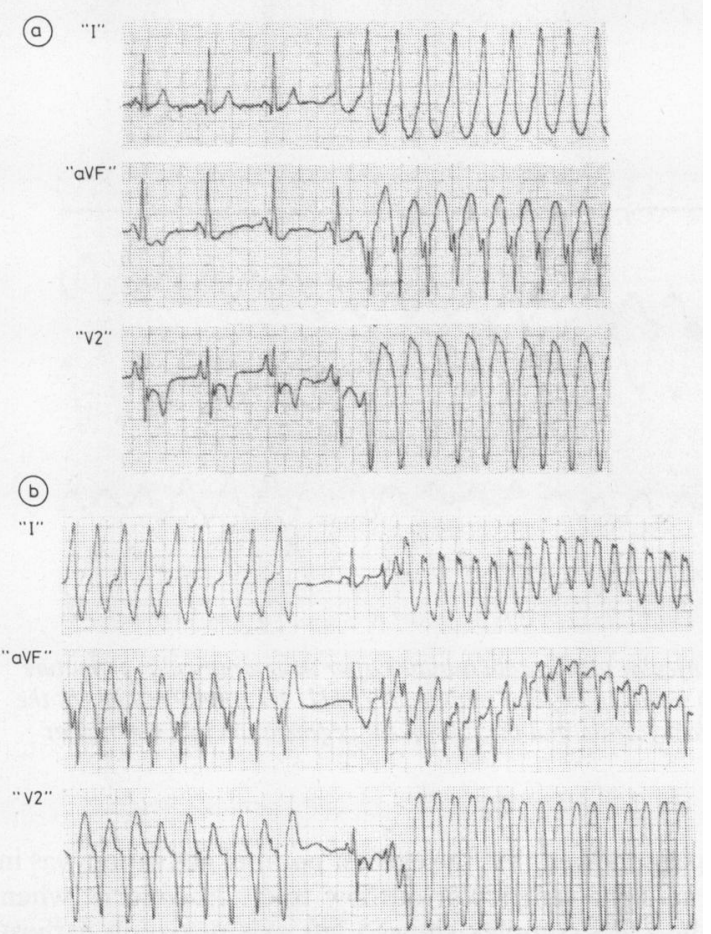

Fig. 3 Three channel ambulatory electrocardiogram showing the onset of ventricular tachycardia (case 2). The arrangement of the electrodes allows three approximately orthogonal bipolar leads to be monitored: a horizontal lead resembling lead I (" $I$ "), an inferior lead resembling lead $a V F$ (" $a V F$ "), and an anterior lead resembling lead V2 ("V2") (paper speed $25 \mathrm{~mm} / \mathrm{s}$ ). (a) Three sinus beats are followed by a fusion beat, which is followed in turn by sustained ventricular tachycardia. (b) After 10 minutes ventricular tachycardia stops spontaneously. After one sinus beat ventricular tachycardia recurs at a faster rate. This eventually deteriorated into ventricular fibrillation from which the patient was resuscitated.

\section{ECHOCARDIOGRAPHY}

In all patients echocardiograms showed an abnormally dilated right ventricular cavity. There were no abnormalities of the tricuspid valve apparatus to suggest Ebstein's anomaly, and in all cases left ventricular cavity size and overall systolic function appeared normal.

\section{ANGIOGRAPHY}

Right ventricular angiography showed an enlarged cavity and impaired contractility in all patients (Table). There was global akinesis in three patients, while the remainder showed regional abnormalities in wall motion. In these two patients one or more areas (inferior, apical, outflow tract) were hypokinetic or akinetic, but in none was there evidence of diverticula. Despite these findings there was only one patient who had a raised right ventricular end diastolic pressure. Left ventricular angiography was nor$\mathrm{mal}$ in all patients as were left ventricular pressures. In both patients in whom coronary angiography was performed coronary anatomy was normal.

Left ventricular radionuclide angiograms confirmed the normal left ventricular function and showed ejection fractions within the normal limits for our laboratory in all patients (Table). Selective right ventricular radionuclide angiography again indicated the abnormalities in right ventricular structure and function. All patients had dilatation of the right ventricular cavity, and, although there were apparent regions of differential contractile abnormalities, it was not possible to localise them with accuracy. There were no diverticula seen on right ventricular radionuclide angiography. The calculated ejection fraction was impaired in all cases, being considerably below the normal range for our laboratory $(0.48 \pm 0.06)$.

\section{ELECTROPHYSIOLOGICAL TESTS}

All patients underwent electrophysiological testing; in two ventricular tachycardia occurred spontaneously (Fig. 4), and in all it could be induced by program-

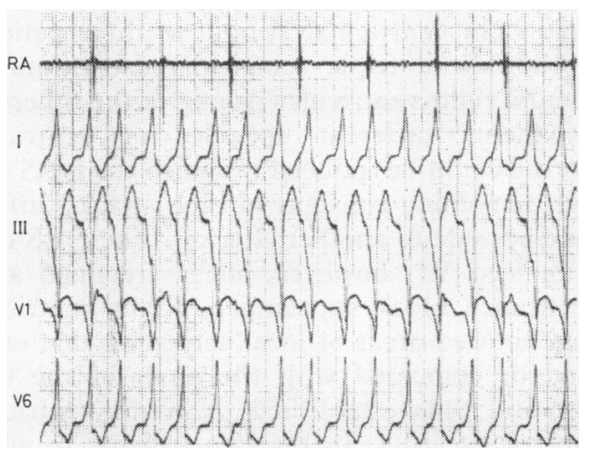

Fig. 4 Right atrial (RA) electrogram confirming atrioventricular dissociation during spontaneous ventricular tachycardia (case 2). Leads I, III, VI, and V6 are shown (paper speed $25 \mathrm{~mm} / \mathrm{s}$ ). 

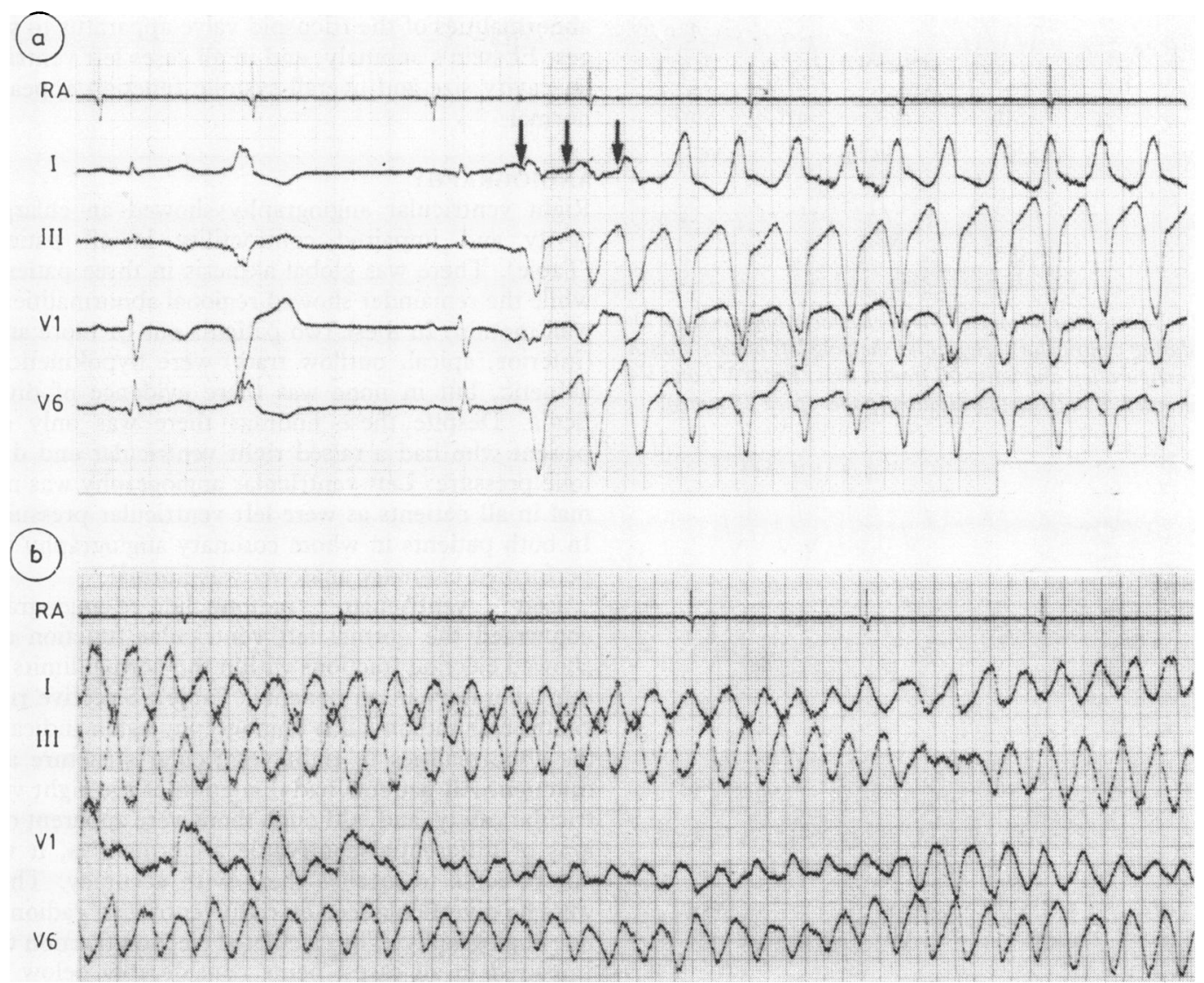

Fig. 5 Electrocardiograms (case 5) showing (a) sustained ventricular tachycardia initiated after three ventricular premature stimuli delivered during sinus rhythm; right atrial activity $(R A)$ is shown together with leads I, III, VI, and V6; and (b) the ventricular rate accelerating two minutes after the initiation of tachycardia and the subsequent degeneration into ventricular fibrillation (paper speed $50 \mathrm{~mm} / \mathrm{s}$ ). Cycle length $=280 \mathrm{~ms}$.

med electrical stimulation. The mode of initiation was by single right ventricular stimuli in three patients, and by double or triple ventricular extrastimuli or incremental right ventricular pacing in the others. In three patients different episodes of ventricular tachycardia could be induced in which the QRS axis, although remaining constant in each attack, differed from one episode to another (Fig. 5). The QRS complexes in lead V1, however, always retained a left bundle branch block appearance. Right ventricular mapping showed areas of local activation that either preceded or coincided with the onset of the QRS complex and thus established the right ventricular origin of tachycardia. In the two patients in whom activation coincided with the onset of $Q R S$, left ventricular mapping was performed and failed to indicate earlier activation of the left ventricle. Where the frontal axis was directed inferiorly right ventricular map- ping showed that the earliest point of activation was in the right ventricular outflow tract. In contrast when the QRS complex showed left axis deviation earliest ventricular activation occurred in the inferolateral region.

In two patients the initiation of ventricular tachycardia was quickly followed by degeneration into ventricular flutter and then ventricular fibrillation (Fig. 5), from which they were defibrillated promptly and without consequence. One had experienced palpitations associated with syncope before study, but interestingly neither patient had required resuscitation from ventricular fibrillation previously. The remaining patients all maintained full consciousness during sustained tachycardia, which could be terminated by either single or double ventricular extrastimuli or overdrive pacing.

In one patient who showed different episodes with 


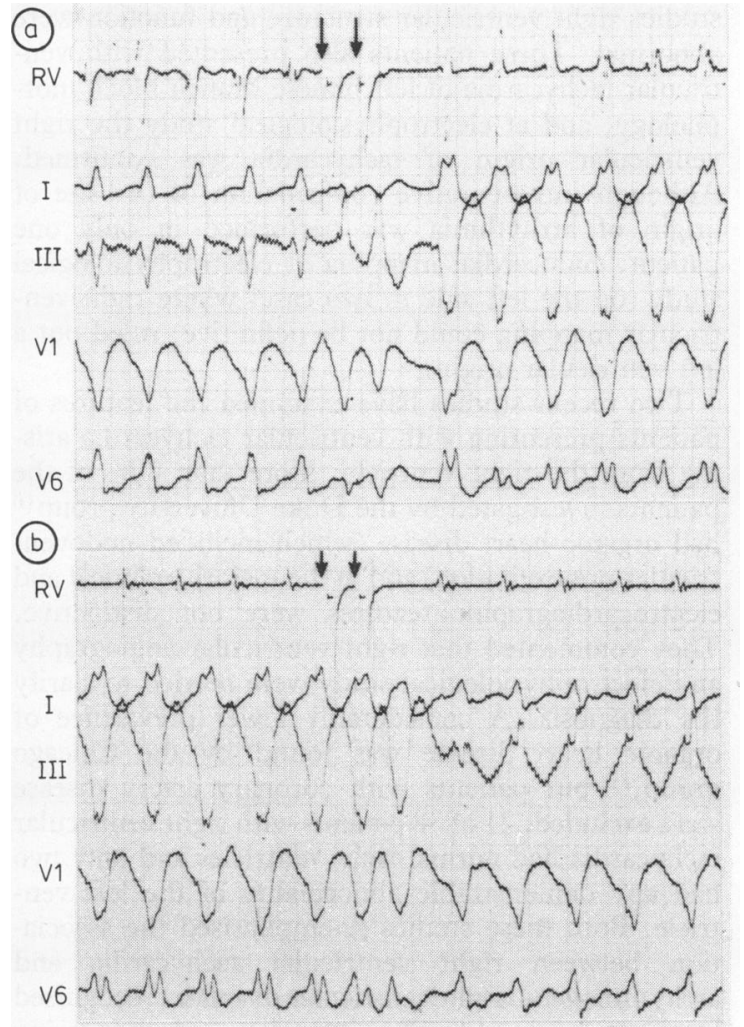

Fig. 6 Electrocardiograms (case 2): multiple morphologies of sustained ventricular tachycardia showing persistence of left bundle branch block configuration but different frontal $Q R S$ axes. Right ventricular $(R V)$ electrogram is shown together with leads I, III, VI, and V6 (paper speed $50 \mathrm{~mm} / \mathrm{s}$ ). (a) Ventricular tachycardia with horizontal $Q R S$ axis is interrupted by two ventricular premature stimuli (arrows) but continues with an extreme leftward frontal axis. (b) Two ventricular premature stimuli (arrows) delivered during ventricular tachycardia with extreme left axis deviation are converted into tachycardia with an inferior $Q R S$ axis. Note the occurrence of the ventricular electrogram during apparent electrical diastole during the left axis tachycardia and the similarity between the $Q R S$ complexes during tachycardia and the paced complexes.

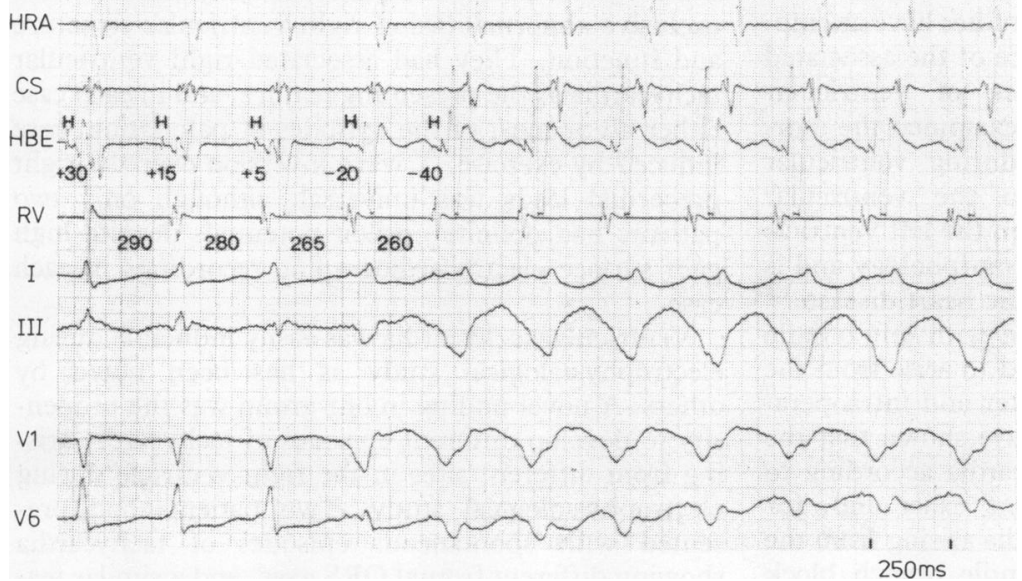

Fig. 7 Electrocardiogram (case 2) showing atrioventricular nodal re-entry tachycardia followed by the spontaneous appearance of ventricular tachycardia. Four intracardiac electrograms (HRA, high right atrium; $C S$, coronary sinus; $H B E$, His bundle; $R V$, right ventricle) are shown with leads $I, I I I, V 1$, and V6 (paper speed $100 \mathrm{~mm} / \mathrm{s}$ ). The first beat shows a narrow $Q R S$ complex during atrioventricular nodal re-entry tachycardia with atrial and ventricular activity occurring simultaneously. The next three complexes show progressive fusion and shortening of the HV interval (ms) as the ventricular rate accelerates from 290 to $260 \mathrm{~ms}$ and ventricular tachycardia becomes established with the fifth complex of the trace. 
three different QRS patterns, endocardial mapping during one episode showed local electrical activation during apparent electrical diastole and preceding the onset of the QRS complex by $75 \mathrm{~ms}$ (Fig. 6). Stimulation at this site produced QRS complexes identical in appearance to those of the tachycardia. This patient also had episodes of narrow QRS complex supraventricular tachycardia which were shown to be due to atrioventricular nodal re-entry. During induced atrioventricular re-entry tachycardia there were short non-sustained episodes of ventricular tachycardia during which the onset of ventricular activity preceded the His bundle deflection (Fig. 7). At no time during premature atrial stimulation or His bundle stimulation was there evidence of accessory nodoventricular or fasciculoventricular conduction. Similarly, the absence of a left bundle branch morphology with atrial pacing excluded the presence of nodoventricular or nodofascicular fibres in the other patients. ${ }^{18}$

\section{FOLLOW UP}

The patients have been followed up for 9-57 (mean 31) months. To date one patient remains asymptomatic taking disopyramide; in two patients the tachycardia is controlled with amiodarone, having proved refractory to conventional antiarrhythmic drugs, and in one patient it is controlled with a combination of amiodarone and propafenon (Table). The remaining patient was refractory to various antiarrhythmic drugs and underwent excision of an area of the right ventricular outflow tract: in the four months of follow up she has remined free from palpitation.

\section{Discussion}

Ventricular tachycardia has for many years been recognised in association with various cardiac disorders but has also been known to occur when there is no apparent heart disease. ${ }^{12}$ Several studies have attempted to relate the clinical importance of the associated heart disease with the presence of ventricular tachycardia. Additional work has examined the morphology of the QRS complex during ventricular tachycardia and related this to the ventricular origin. ${ }^{347}$ Tachycardia arising from the left ventricle is characterised by a right bundle morphology and is frequently associated with ischaemic heart disease. ${ }^{48}$ In contrast ventricular tachycardia with left bundle branch morphology was considered to arise from the right venticle. ${ }^{4}$ Endocardial catheter and intraoperative mapping studies, however, have shown that the classification of ventricular tachycardia according to QRS morphology is by no means exact and, for instance, that ventricular tachycardia arising from the left ventricle may have a left bundle branch block morphology. ${ }^{519}$ Our series of patients was selected on the basis that by angiographic and echocardiographic studies right ventricular structure and function were abnormal. These patients also presented with ventricular tachycardia of left bundle branch block morphology, and at electrophysiological study the right ventricular origin of tachycardia was confirmed. Although intraoperative confirmation of the site of origin of arrhythmia was performed in only one patient, endocardial mapping at electrophysiological study (on the left side in two cases where right ventricular mapping could not be definitive) ruled out a left ventricular origin.

Two recent studies have examined the features of patients presenting with ventricular tachycardia arising from the right ventricle. More than $80 \%$ of the patients investigated by the Duke University group ${ }^{10}$ had organic heart disease (which included nodoventricular pre-excitation) and in the majority clinical and electrocardiographic features were not distinctive. They commented that right ventricular angiography and electrophysiological study were needed to clarify the diagnosis. A considerably lower prevalence of organic heart disease was found by the Chicago group, ${ }^{11}$ but patients with coronary artery disease were excluded: 21 of 38 patients with right ventricular tachycardia had normal right ventricles and only two had any demonstrable abnormality of the left ventricle. Both these studies re-emphasised the association between right ventricular tachycardia and arrhythmogenic right ventricular dysplasia recognised by Fontaine et al. 9 The extensive and pioneering experience of the Paris group with this condition has been summarised together with a review of published reports. ${ }^{20}$ The clinical features and nature of the tachycardia in our patients were precisely the same as those in the patients described by Fontaine as having "arrhythmogenic right ventricular dysplasia." Our patients were young and predominantly male and had exclusive abnormalities of right ventricular structure and function. They had associated right ventricular tachycardia as the presenting feature, and in each case either all or most of the episodes of palpitation were induced by exercise. $T$ wave inversion over the right precordial leads was universal, although only two patients had definite epsilon potentials despite high gain surface electrocardiographic recordings in each case.

Ventricular tachycardia was easily inducible during electrophysiological study as has been noted by others. A novel finding in our group was the propensity to develop different episodes of tachycardia arising from different sites in the right ventricle during electrophysiological study. Two patients had presented with spontaneous episodes of tachycardia showing different frontal QRS axes, and a similar feature has been noted by Reiter et al. ${ }^{10}$ Operative map- 
ping was not, however, performed during different morphological forms of ventricular tachycardia, and different exit points from the same area of the right ventricle cannot be entirely excluded. Supportive evidence for different origins is suggested by the different sites of earliest activation during endocardial mapping and the notably different frontal QRS axis during the separate episodes. Clearly, this has important implications in those patients who require operative treatment. Both Fontaine et al..$^{21}$ and Reiter et al. ${ }^{10}$ commented on patients in their series who underwent apparently successful cryoablation for ventricular tachycardia only to have ventricular tachycardia with a different morphology re-emerge later. The apparent instability of the ventricular arrhythmia, perhaps reflecting the widespread nature of the right ventricular disturbance, was suggested by the propensity, shown in all cases, for ventricular fibrillation; in three patients it occurred while out of hospital. Although in the other two it occurred during the electrophysiological study, in one patient it followed the induction of the identical morphology of ventricular tachycardia with which the patient presented. In the other patient ventricular fibrillation followed the use of three premature ventricular stimuli used in an attempt to terminate sustained ventricular tachycardia. Although there were no deaths in our series and all patients were treated after their electrophysiological investigation, these features do not suggest a benign outlook in the absence of effective antiarrhythmic treatment. Previous authors have observed a small incidence of sudden death in right ventricular dysplasia, 1020 and the accompanying report from this institution suggests that the prognosis is not benign. ${ }^{12}$

Althought right ventricular angiography showed the dilated and poorly contractile right ventricle, the abnormalities were also detected on cross sectional echocardiography and multigated radionuclide angiography. These latter techniques may prove to be of greater value in determining the extent of the disease and documenting progress.

Some aspects of the clinical importance of right ventricular tachycardia have been elucidated recently. ${ }^{101120}$ Our patients appear to have had several adverse features that necessitated vigorous antiarrhythmic treatment. Drug treatment has proved effective in most of our patients, while the potential for operative treatment has been shown in the other. During follow up no symptoms or signs have appeared that suggest either progression of right ventricular dysfunction or the appearance of left ventricular involvement. Repeat cardiac catheterisation has not, however, been performed. Two patients (cases 3 and 4) had histological examination of right ventricular myocardium. In the patient who underwent opera- tion the features were similar to those found in other cases of right ventricular dysplasia. ${ }^{22}$ The other patient had the biopsy specimen obtained during catheterisation from an area of the right ventricle that was contracting poorly. Examination showed hypertrophied fibres with interstitial fibrosis, features which were consistent with a diagnosis of dilated (congestive) cardiomyopathy. These findings illustrate some aspects of the difficulty of defining the pathogenesis of the right ventricular disease and predicting the prognosis.

Whether a diagnosis of right ventricular dysplasia or dilated cardiomyopathy that selectively affects the right ventricle has different prognostic implications remains to be answered. The mean age of our patients was younger than many published series of patients with right ventricular dysplasia. It is of interest, however, that in older patients with right ventricular dysplasia left ventricular abnormalities have been seen and this suggests that the potential for extension of the disease exists. ${ }^{23} 24$

In patients with ventricular tachycardia of left bundle branch configuration and normal left ventricles diligent evaluation of right ventricular structure and function is required, particularly when $T$ waves are inverted in the right precordial leads. This may allow the adverse prognostic features of this group to be distinguished from those in other patients in whom both ventricles are normal and who in the absence of organic heart disease appear to have a benign prognosis. ${ }^{411}$

\section{References}

1 Froment R, Gallavardin L, Cahen P. Paroxysmal ventricular tachycardia. A clinical classification. Br Heart $\mathcal{f}$ 1953; 15: 172-8.

2 Chapman JH, Schrank JP, Crampton RS. Idiopathic ventricular tachycardia: an intracardiac electrical, hemodynamic and angiographic assessment of six patients. Am ₹ Med 1975; 59: 470-80.

3 Bisteni A, Sodi-Pallares D, Medrano GA, Pileggi F. A new approach for the recognition of ventricular premature beats. Am f Cardiol 1960; 5: 358-69.

4 Pietras RJ, Mautner R, Denes $P$, et al. Chronic recurrent right and left ventricular tachycardia: comparison of clinical, hemodynamic and angiographic findings. Am $\mathcal{J}$ Cardiol 1977; 40: 32-7.

5 Josephson ME, Horowitz LN, Farshidi A, Spielman SR, Michelson EL, Greenspan AM. Recurrent sustained ventricular tachycardia. 4. Pleomorphism. Circulation 1979; 59: 459-68.

6 Josephson ME, Horowitz LN, Farshidi A, Spear JF, Kastor JA, Moore EN. Recurrent sustained ventricular tachycardia. 2. Endocardial mapping. Circulation 1978; 57: 440-7. 
7 Manning GW, Ahuja SP, Guttierrez MR. Electrocardiographic differentiation between ventricular ectopic beats from subjects with normal and diseased hearts. Acta Cardiol (Brux) 1968; 23: 462-70.

8 Bodenheimer MM, Banka VS, Helfant RH. Relation between the site of origin of ventricular premature complexes and the presence and severity of coronary artery disease. Am $\mathcal{F}$ Cardiol 1977; 40: 865-9.

9 Fontaine G, Guiraudon G, Frank R, et al. Stimulation studies and epicardial mapping in ventricular tachycardia; study of mechanisms and selection for surgery. In: Kulbertus HE, ed. Reentrant arrhythmias: mechanisms and treatment Lancaster: MTP, 1977: 334-50.

10 Reiter MJ, Smith WM, Gallagher JJ. Clinical spectrum of ventricular tachycardia with left bundle branch morphology. Am $\mathcal{F}$ Cardiol 1983; 51: 113-21.

11 Pietras RJ, Lam W, Bauernfeind R, et al. Chronic recurrent right ventricular tachycardia in patients without ischemic heart disease: clinical, hemodynamic and angiographic findings. Am Heart $\mathcal{F}$ 1983; 105: 357-66.

12 Fitchett DH, Sugrue DD, MacArthur CG, Oakley CM. Right ventricular congestive cardiomyopathy. Br Heart $\mathcal{f}$ 1984; 51: 25-9.

13 Matsumoto M, Matsuo H. Echocardiography for the evaluation of the tricuspid valve, right ventricle and atrium. Prog Cardiovasc Dis 1978; 21: 1-42.

14 Gentzler RD, Briselli M, Gault JH. Angiographic estimation of right ventricular volume in man. Circulation 1974; 50: 324-30.

15 Sugrue DD, Kamal S, Deanfield JE, et al. Assessment of right ventricular function and anatomy using peripheral vein infusion of Krypton $81^{\mathrm{m}}$. Br $\mathcal{F}$ Radiol 1983; 56: 657-63.

16 Curry PVL. Fundamentals of arrhythmias: modern methods of investigation. In: Krikler DM, Goodwin JF, eds. Cardiac arrhythmias. The modern electrophysiological approach. London, Philadelphia, Toronto: WB Saunders, 1975: 39-80.

17 Frank R, Fontaine G, Vedel J, et al. Electrocardiologie de quatre cas de dysplasie ventriculaire droite arythmogène. Arch Mal Coeur 1978; 71: 963-72.

18 Ward DE, Camm AJ, Spurrell RAJ. Ventricular preexcitation due to anomalous nodo-ventricular pathways: report of 3 patients. Eur $\mathcal{F}$ Cardiol 1979; 9: 111-27.

19 Kaplinsky E, Ogawa S, Kmetzo J, Dreifus LS. Origin of so-called right and left ventricular arrhythmias in acute myocardial ischemia. Am $\mathcal{F}$ Cardiol 1978; 42: 774-80.

20 Marcus FI, Fontaine GH, Guiraudon G, et al . Right ventricular dysplasia: a report of 24 adult cases. Circulation 1982; 65: 384-98.

21 Fontaine G, Guiraudon G, Frank R, Vedel J, Grosgogeat $\mathrm{Y}$, Cabrol C. Modern concepts of ventricular tachycardia. Eur $\mathcal{F}$ Cardiol 1979; 8: 565-80.

22 Fontaine G, Guiraudon G, Frank R, et al. Dysplasie ventriculaire droite arythmogene et maladie de Uhl. Arch Mal Coeur 1982; 75: 361-71.

23 Cherrier F, Floquet J, Cuillière M, Neimann JL. Les dysplasies ventriculaires droites. A propos de 7 observations. Arch Mal Coeur 1979; 72: 766-73.

24 Halphen Ch, Beaufils PL, Azancot I, Baudouy P, Manne B, Slama R. Tachycardies ventriculaires récidivantes par dysplasie ventriculaire droite. Association à des anomalies du ventricule gauche. Arch Mal Coeur 1981; 74: 1113-8.

Requests for reprints to Dr Edward Rowland, Division of Cardiovascular Disease, Royal Postgraduate Medical School, Hammersmith Hospital, Du Cane Road, London W12 0HS. 